\title{
Transcriptome and metabolome profiling provide insights into molecular mechanism of pseudostem elongation in banana
}

\author{
Guiming Deng ${ }^{1,2,3^{*}}$, Fangcheng Bi ${ }^{1,2,3}$, Jing Liu ${ }^{4}$, Weidi He ${ }^{1,2,3}$, Chunyu Li ${ }^{1,2,3}$, Tao Dong ${ }^{1,2,3}$, Qiaosong Yang ${ }^{1,2,3}$, \\ Huijun Gao ${ }^{1,2,3}$, Tongxin Dou ${ }^{1,2,3}$, Xiaohong Zhong ${ }^{4}$, Miao Peng ${ }^{4}$, Ganjun $\mathrm{Yi}^{1,2,3}$, Chunhua $\mathrm{Hu}^{1,2,3}$ and Ou Sheng ${ }^{1,2,3}$
}

\begin{abstract}
Background: Banana plant height is an important trait for horticultural practices and semi-dwarf cultivars show better resistance to damages by wind and rain. However, the molecular mechanisms controlling the pseudostem height remain poorly understood. Herein, we studied the molecular changes in the pseudostem of a semi-dwarf banana mutant Aifen No. 1 (Musa spp. Pisang Awak sub-group ABB) as compared to its wild-type dwarf cultivar using a combined transcriptome and metabolome approach.
\end{abstract}

Results: A total of 127 differentially expressed genes and 48 differentially accumulated metabolites were detected between the mutant and its wild type. Metabolites belonging to amino acid and its derivatives, flavonoids, lignans, coumarins, organic acids, and phenolic acids were up-regulated in the mutant. The transcriptome analysis showed the differential regulation of genes related to the gibberellin pathway, auxin transport, cell elongation, and cell wall modification. Based on the regulation of gibberellin and associated pathway-related genes, we discussed the involvement of gibberellins in pseudostem elongation in the mutant banana. Genes and metabolites associated with cell wall were explored and their involvement in cell extension is discussed.

Conclusions: The results suggest that gibberellins and associated pathways are possibly developing the observed semi-dwarf pseudostem phenotype together with cell elongation and cell wall modification. The findings increase the understanding of the mechanisms underlying banana stem height and provide new clues for further dissection of specific gene functions.

Keywords: Auxin efflux carrier proteins, Banana Pseudostem, Dwarfism, Ethylene response factors, Gibberellins

\section{Background}

In banana (Musa spp.) production, plant architecture offers unique horticultural practices including, flower removal, bunch management, controlling pests and diseases, etc. High banana pseudostem is easily broken in a typhoon and needs additional input cost for

\footnotetext{
* Correspondence: guimingdeng2014@163.com

'Institute of Fruit Tree Research, Guangdong Academy of Agricultural Sciences, Guangzhou, China

${ }^{2}$ Key Laboratory of South Subtropical Fruit Biology and Genetic Resource Utilization, Ministry of Agriculture, Guangzhou, China

Full list of author information is available at the end of the article
}

propping. Productivity optimization could be achieved by manipulating banana plant height. Semi-dwarf banana cultivars show good resistance to damages caused by heavy wind and rain [1]. Furthermore, the yield gain associated with shorter stems is also linked to an increased harvest index [2]. In spite of the basic and strategic importance of semi-dwarf stem, the progress on genetic manipulation of banana plant height has been comparatively limited, partially because the ontogenesis of the pseudostem is not as easily characterized as for other organs, and its external morphological complexity

(c) The Author(s). 2021 Open Access This article is licensed under a Creative Commons Attribution 4.0 International License, which permits use, sharing, adaptation, distribution and reproduction in any medium or format, as long as you give appropriate credit to the original author(s) and the source, provide a link to the Creative Commons licence, and indicate if changes were made. The images or other third party material in this article are included in the article's Creative Commons licence, unless indicated otherwise in a credit line to the material. If material is not included in the article's Creative Commons licence and your intended use is not permitted by statutory regulation or exceeds the permitted use, you will need to obtain permission directly from the copyright holder. To view a copy of this licence, visit http://creativecommons.org/licenses/by/4.0/. The Creative Commons Public Domain Dedication waiver (http://creativecommons.org/publicdomain/zero/1.0/) applies to the data made available in this article, unless otherwise stated in a credit line to the data. 
is not so attractive as compared to leaves, roots, and flowers [3].

In vitro techniques are often applied in the banana industry for producing large-scale disease-free plantlets with higher yield potential. However, as a result of such processing, somaclonal variations often appear, which are considered as one of the major disadvantages for such techniques [4]. Dwarfism and giant variants are often observed in banana tissue culture plantlets $[1,5,6]$. On the other hand, the variants derived from tissue culture processing, however, are a good source of research materials suitable for a comparative transcriptome and metabolome analysis. Pisang Awak (Musa spp.) is a cultivated banana subgroup with high yield and superior taste quality, which is widely grown in Asian countries as a desert fruit but for juice/brewing in African countries [7]. Normally, the plants of Pisang Awak varieties are vigorous with a height around 4.5-5.5 m. In our Musa collections, there is a dwarf Pisang Awak variety 'Aifen No. 1' (wild type, WT), of which the height is around $2.0-2.5 \mathrm{~m}$. Recently, a semi-dwarf mutant (MT) with the height $2.8-3.5 \mathrm{~m}$, was identified by screening the somaclonal variations from 'Aifen No. 1' tissue cultured plants. Previous studies showed that hormone biosynthesis pathways are responsible for such variations [1].

So far, the key pathways that have been associated with the stem height are the gibberellic acid (GA) metabolism and signaling, elasticity of the cell-wall of expanding cells, and cross-talk between GA and other hormone metabolism and signaling pathways i.e. auxin, brassinosteroids (BR), and abscisic acid (ABA) [3]. Chen et al., [1] studied a dwarf mutant of 'Williams' banana variety and demonstrated the role of GA in dwarfism. In peach trees, the brachytic dwarfism trait $(d w)$ was associated with a nonsense mutation in a GA receptor PpeGID1c [8]. GA biosynthesis pathway-related enzymes such as ent-Copalyl diphosphate synthase, ent-Kaurene synthase, ent-Kaurene 19-oxidase, GA12-aldehyde 7oxidase, GA 20-oxidase, GA 3 3 -hydroxylase, and GA 2oxidase have been successfully identified in Arabidopsis, pumpkin, and runner bean [9]. A previous study on GA metabolisms between Williams banana and its dwarf mutant shed light on the GA regulated stem development in banana and identified six main genes regulating differential GA content [1]. While this study focused on GA metabolismrelated genes, the other pathways related to stem elongation remain poorly explored $[9,10]$.

In plants, the stem is initiated at shoot apical meristem (SAM) and in the case of seed plants, it is originated from the sub-apical region (called rib zone, RZ) of SAM [11]. Additionally, the monocot stem elongation is promoted by intercalary meristems (IM). Both RZ and IMs are the main sites where stem growth starts under GA stimuli [3]. GA degrades DELLA proteins and stimulates the cell division in RZ zone. DELLAs, the proteins controlling cell division in the stem, are functionally associated with transcription factors (TFs) e.g. DELLAs bind and inhibit the activity of class 1 teosinte branched 1 (TCP), cycloidea, and proliferating cell factor TFs, which further activates other genes associated with cell cycle progression in stem [12]. Recent studies have highlighted the direct link of DELLAs and cell prolification in RZ, which not only influences stem elongation but also the SAM size. Other than DELLAs, organ boundary genes such as lateral organ boundaries (LOB) domaincontaining genes, have also been implicated in stem elongation by restricting signals that control stem elongation [13]. The establishment of vascular pattern in the early stages of stem development is a key stage and is under the influence of auxin flow. In this regard, the role of auxin efflux carrier proteins has been associated with the cell elongation in root epidermal cells but such a role in stem elongation has not been explored yet [14]. After the initial growth based on cell prolification, cell expansion comes into action for internode growth. Stem growth is adjusted to changeable conditions by the rapid differential growth. For cell prolification as well as expansion, the prominent role of cell-wall related genes is also an important consideration. Finally, the coordination between tissue layers through signaling also plays an important role during stem elongation. The role of auxin in BR production and synthesis as well as the signaling between $B R$ and very long-chain fatty acids in the epidermis are good examples $[15,16]$.

With the availability of the banana genome, it is now possible to gain a deeper insight into the mechanisms governing plant height [17-19]. Unbiased modern highthroughput technologies such as whole transcriptome and metabolome have geared up the discovery of genetic factors controlling different traits in plants including, biotic and abiotic stresses, and agronomic traits [20-23]. Furthermore, the combination of multiple omics techniques has resulted in the elucidation of different pathways in banana such as peel ripening and the effect of melatonin on delaying anthracnose incidence [24-26]. In this study, we aimed at identifying the differentially expressed genes and metabolites in pseudostems of Pisang Awak Aifen No. 1 dwarf wild type and its semidwarf mutant. The transcriptome and metabolome responses involved hormonal signaling including GA, auxin, BR, ABA, and ethylene, and cell prolification and elongation.

\section{Results}

\section{Plant phenotype}

In the field, the adult dwarf wild type (WT) banana Aifen No.1 (Musa spp. ABB group) plant presented 
short pseudostems ranging from $2.0-2.5 \mathrm{~m}$ with the stem girth ranging from $95.5-98.5 \mathrm{~cm}$. In addition, the bunch weight of WT was $15-20 \mathrm{~kg}$. The identified Aifen No.1 mutant (MT) plant had a semi-dwarf stature with $2.8-3.5 \mathrm{~m}$ plant height, $92.5-95.0 \mathrm{~cm}$ stem girth, and a relatively heavier bunch i.e. $25-30 \mathrm{~kg}$ (Table 1; Fig. 1a\&b).

\section{Transcriptome analysis}

We investigated the changes in gene expression profiles in the pseudostems of WT and MT plants. With three biological replicates, the transcriptome sequencing of the six samples yielded a total of $56.78 \mathrm{~Gb}$ clean data with an average of $94.46 \%$ bases scoring Q30 (Additional Table 1). Of the total clean reads, 83.68 to $90.25 \%$ were unique matches with Musa genome (Musa acuminata DH Pahang V2 and Musa balbisiana DH PKW) $[18,27]$. Overall, the Fragments Per Kilobase of Transcript per Million Fragments Mapped (FPKM) for MT was higher as compared to WT, when FPKM >1 was used as a threshold to determine the gene expression (Fig. 2a). Pearson correlation coefficient (PCC) between WT and MT replicates ranged from 0.25 to 0.87 (Additional Fig. 1). This is expected since MT was obtained from WT by tissue culture and probably, only a few genes were mutated. We also observed that there is a high intra-varietal diversity for pseudostem transcriptome. Therefore, we would recommend more replicates in future studies related to pseudostem growth.

\section{Differentially expressed genes between WT and MT}

DESeq2 analysis revealed that a total of 127 genes were differentially expressed (DEG) between WT and MT; 25 and 102 DEGs showed decreased and increased expression in MT as compared to WT, respectively. These results imply that very few genes are involved in pseudostem height in banana. The gene expression cluster analysis based heatmap of each differential group is presented in Additional Fig. 2. Of the DEGs, only five genes were exclusively expressed in WT. The NR annotation showed that these genes were predicted as BIG GRAIN 1like E isoform X1 (gene-C4D60_Mb04t03190), protein SRG1-Like (gene-C4D60_Mb11t04180), S-norcoclaurine synthase 1-like (gene-C4D60_Mb11t04200), an uncharacterized protein (Ma02_g04590), and a stellacyanin isoform X1 (Ma05_g16810) (Additional Table 3). Only one gene (gene-C4D60_Mb07t24780) was exclusively expressed in MT banana with a $\log 2$ fold value of 7.082 . This gene was predicted as an integral part of the membrane. The Kyoto Encyclopedia of genes and Genomes (KEGG) analysis revealed that the top-five key biological pathways that were significantly enriched in WT vs MT banana were metabolic pathways, biosynthesis of secondary metabolites, ribosomes, tyrosine metabolism, and phenylpropanoid biosynthesis (Fig. 2b; Additional Fig. 3).

\section{Differentially expressed genes related to plant hormones} Regulation of GAs causes banana plants to exhibit the dwarf phenotype [1]. Hence, it is important to check if the expression of GA biosynthesis pathway-related genes were differentially modulated between WT and MT. We found that an $\mathrm{N}$-acetylglucosaminyl-phosphatidylinositol biosynthetic protein gpi1-like (gibberellin-regulated protein 6) (Ma06_g04390) was highly up-regulated in MT as compared to WT. We also observed a probable 2oxoglutarate-dependent dioxygenase (Ma07 g15430) which was up-regulated in MT. Owing to cross-talk between BR and GAs, we looked for expression of related genes in our DEGs and observed the upregulation of two BRI genes (gene-C4D60_Mb10t18040 and Ma10_g11870). Because LOB domain-containing genes are targeted by DELLAs, the decrease in the expression of one LOB domain-containing gene was

Table 1 Variation in stem height and stem girth at different time points of studied WT and MT Aifen No. banana plants

\begin{tabular}{llll}
\hline Time & Trait & Wild type (Dwarf) & Mutant (High) \\
\hline 5-6 leaves seedlings & Stem girth $(\mathrm{cm})$ & $3.5-5.0$ & $2.0-3.0$ \\
& Plant height $(\mathrm{m})$ & $0.12-0.14$ & $0.18-0.22$ \\
Three months & Stem girth $(\mathrm{cm})$ & $20.3-22.5$ & $15.5-18.5$ \\
& Plant height $(\mathrm{m})$ & $0.4-0.8$ & $1.0-1.3$ \\
Six months & Stem girth $(\mathrm{cm})$ & $45.5-49.5$ & $38.5-40.6$ \\
Nine months & Plant height $(\mathrm{m})$ & $1.0-1.2$ & $1.5-2.0$ \\
& Stem girth $(\mathrm{cm})$ & $80.6-82.5$ & $74.5-76.8$ \\
Budding stage & Plant height $(\mathrm{m})$ & $1.6-1.8$ & $2.5-3.0$ \\
Harvest period & Stem girth $(\mathrm{cm})$ & $95.5-98.5$ & $92.5-95.0$ \\
& Plant height $(\mathrm{m})$ & $2.0-2.5$ & $2.8-3.5$ \\
\hline
\end{tabular}




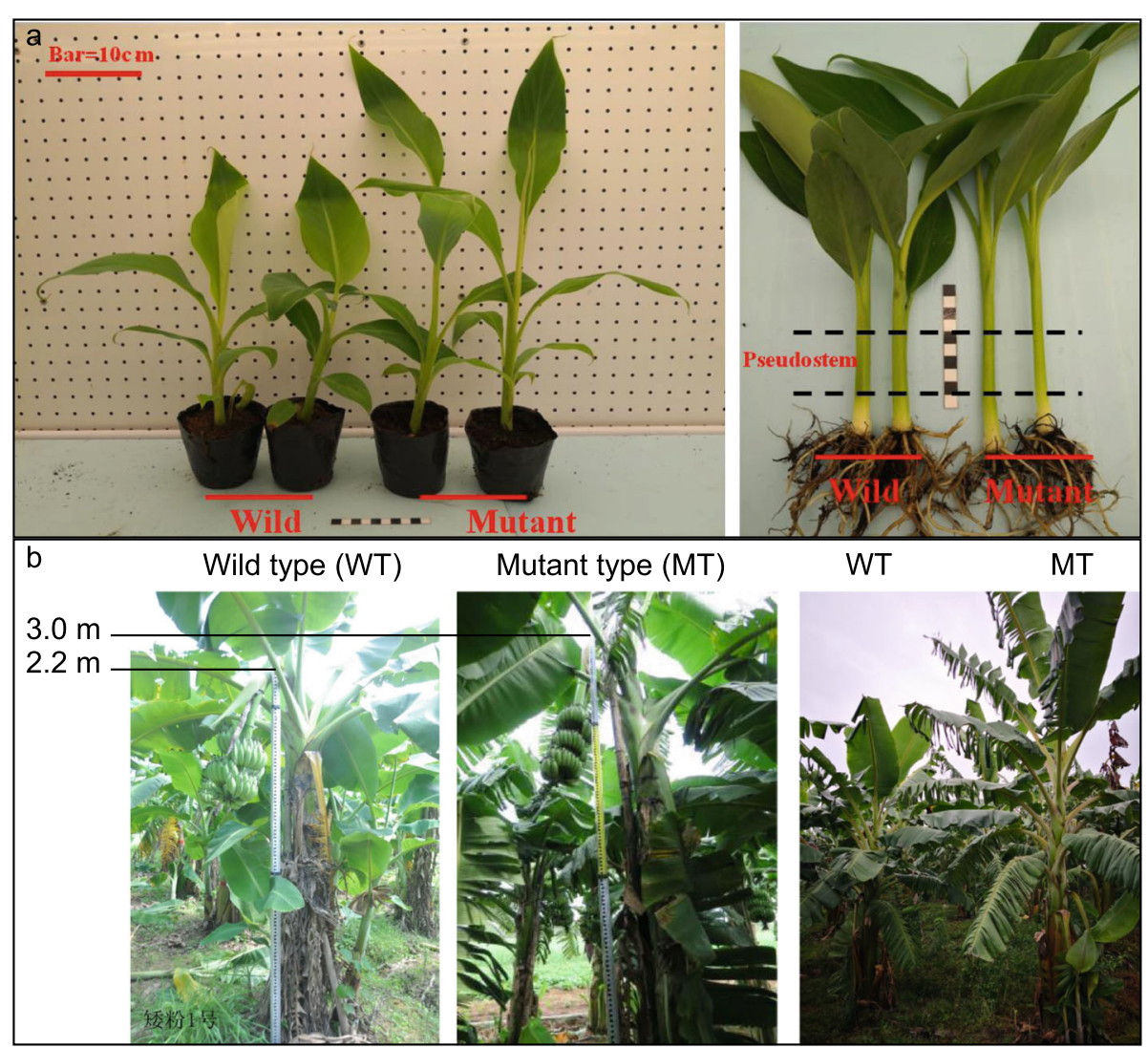

Fig. 1 a) Six-week old wild type and semi-dwarf mutant plants of Aifen No. 1. The second panel shows the pseudostems used for combined omics analysis and $\mathbf{b}$ ) comparison of plant height
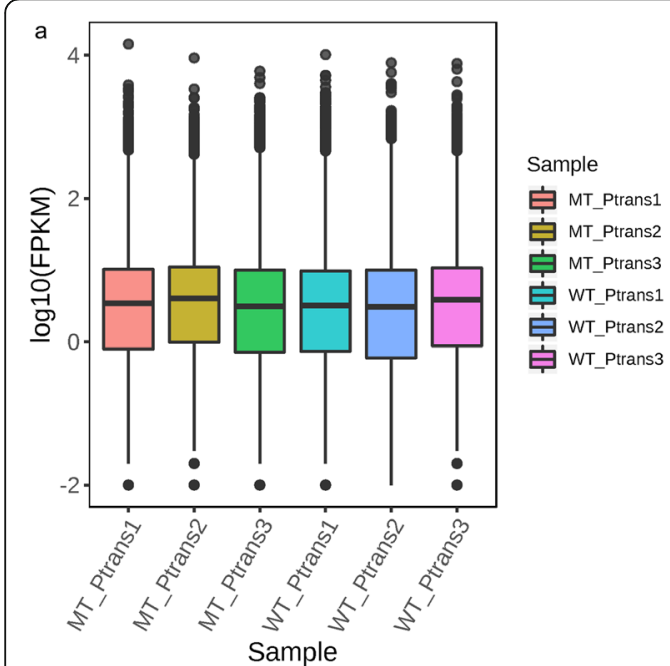

b

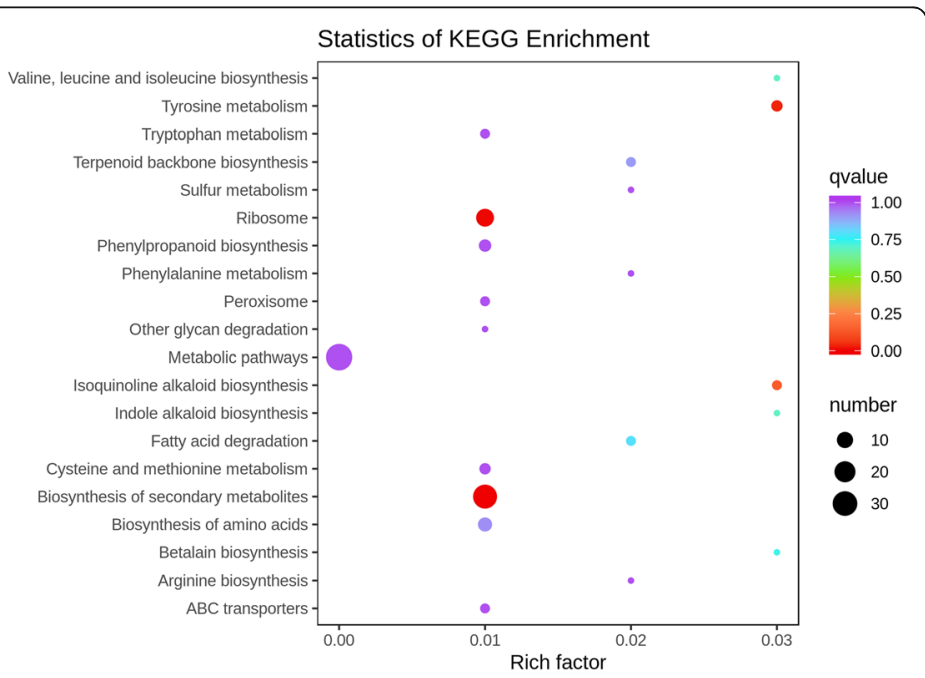

Fig. 2 a Discrete and overall degree of gene expression in the replicates of both WT and MT Aifen No. 1 banana pseudostems. $\mathbf{b}$ Scatter plot of 20 most enriched KEGG pathways between WT and MT Aifen No. 1 banana pseudostems. The degree of enrichment is shown by Rich factor, Qvalue, and the number of genes enriched in each pathway 
differentially expressed [28]. DELLAs interact with TFs such as TCP and Jasmonate ZIM domain-containing proteins (JAZ). In this regard, we found that two genes were up-regulated in MT; one JAZ (gene-C4D60 Mb07t13750) and one TCP (Ma03_g32580). Among the highly expressed genes in MT, the increase in the expression of two interleukin-1 receptor-associated kinase 4 genes (gene-C4D60_Mb08t19920 and gene-C4D60_ Mb10t14420) with fold change values of 172.317 and 93.8, respectively, is an important observation. Previously it is known that plant stature-related receptor-like kinase 2 (PSRK2) acts as a factor that determines stem elongation toward gibberellins response in rice [29].

Mevalonate pathway has a minor contribution to the GA biosynthesis pathway [10]. In this regard, we observed that two GHMP kinase genes (Ma08_g34050 and gene-C4D60_Mb08t33270) having mevalonate kinase activity (based on GO annotation) had increased expression in MT. It is also known that cytosolic acetyl-CoA is further involved in the biosynthesis of mevalonatederived isoprenoids [30]. One gene (gene-C4D60 Mb01t19300) annotated as cytosolic acetyl-CoA showed decreased expression in MT.

Four members of the ethylene responsive factor (ERF) (ERF/AP2) family (Ma02_g02820, Ma05_g04880, Ma09_ g22060, and gene-C4D60_Mb02t03000) showed increased expression in MT as compared to WT. The GCN5-related N-terminal acetyltransferases (GNATs) are directly involved in the transcriptional regulation of meristem regulatory genes such as WUSCHEL [31]. We observed an increased expression of one GNAT (geneC4D60_Mb10t18260) in our transcriptome dataset (Additional Table 3).

In terms of the role of auxin in BR synthesis and signaling, as well as hypocotyl elongation, the expression of two genes (gene-C4D60_Mb10t21290 and Ma10_ g08300) was relatively increased in MT suggesting a role of auxin in banana pseudostem elongation. One of the exclusively expressed genes in WT was BIG GRAIN 1like, which is known to be involved in auxin transport [32]. The expression of a HB-HD-ZIP TF (gene-C4D60_ Mb09t13640) was increased in MT. Our transcriptome data showed that two genes (Ma06_g03830 and geneC4D60_Mb08t08250) belonging to ABC transporter G and $C$ families, respectively, were up-regulated in MT suggesting the involvement of polar auxin transport in stem elongation [33].

Among the highly expressed genes in MT, the second highest expression was noted for a TIFY-5A-like protein (Ma07_g01220). This gene is known for its role as a repressor of Jasmonate responses [34, 35]. Three genes annotated as phospholipase A1-II 5-like showed increased expression in MT (gene-C4D60_ Mb07t09300, Ma02_g10300, and Ma08_g26980).
Phospholipase As are involved in root elongation as well as in auxin signaling [36].

\section{Differentially expressed genes related to Cell Wall, cell growth, and stem height}

A dedicated mechanism involving pectin methylesterase inhibitors (PMEI) controls the cell-wall rigidity and decreases its visco-elasticity [37]. The lower expression of one PMEI (gene-C4D60_Mb03t05630) in MT might be considered a strategy towards stem elongation [38]. We also observed reduced expression of cellulose synthaselike protein (Ma08_g05160) and a member of glycosyltransferase like family 2 (cellulose synthase-like protein E2), while two probable mannan synthases (geneC4D60_Mb07t06270 and Ma07_g22600) showed increased expression in MT (Additional Table 3).

We observed that the expression of a fatty-acyl-CoA synthase was increased in MT banana. Furthermore, the expression of four caffeic acid 3-O-methyltransferases (COMT) (Ma09_g18140, Ma09_g18200, gene-C4D60_ Mb09t20170, and gene-C4D60_Mb09t20150) was increased in MT [39, 40].

We also observed the relatively lower expression of genes related to starch degradation i.e. inactive betaamylase 9 (Ma03_g08740) [41], 1-acyl-sn-glycerol-3phosphate acyltransferase PLS1 which has also been reported to be involved in growth of the cucumber seedlings [42], and a stellacyanin, 3-ketoacyl-CoA synthase 4-like (Ma05_g16810) (key enzyme catalyzing the first reaction in fatty acid elongation and determining the substrate specificity) [43] (Additional Table 3). Apart from hormones and cell-wall structural control of cell elongation, it is known that codeine 3-O-demethylases are linked to plant cell division and stem elongation via the demethylation of mono-, di-, and tri-methylated lysine- 4 residues of histone subunit 3 . We found that a codeine 3-O-demethylase (gene-C4D60_Mb11t04180) had almost no expression in MT [44] (Additional Table 3). GABA accumulation is known to cause cell elongation defects. Our transcriptome data showed exactly this feature where one transmembrane amino acid transporter protein (gene-C4D60_Mb01t32380; probable GABA transporter 1) had lower expression in MT (Additional Table 3) [45]. White-brown complex homolog protein 15 (Ma06_g03830) (ABC transporter) showed increased expression in MT. This gene is highly expressed in developing cotton fiber cells and is correlated with cotton fiber elongation [46].

A probable carboxylesterase 18 was highly expressed in MT as compared to WT (92.9-fold increase in the expression). These genes have been functionally associated with plant height in maize through genome-wide association studies [47]. The 9-cis-epoxycarotenoid dioxygenase (Ma04_g38030) was highly expressed in MT. 
These are implicated in early seedling growth after germination and have shown significantly longer roots and shoots as compared to non-transformed plants [48].

We also found three aminocyclopropanecarboxylate oxidases (ACO) (gene-C4D60_Mb05t08270, Ma05_ g09360, and gene-C4D60_Mb06t13490) that showed increased expression in MT as compared to WT. ACO1 was shown to affect internode elongation at the heading stage in rice [49].

It is known that ribosomal subunit proteins regulate leaf morphology as well as plant architecture [50]. In this regard, we observed that a ribosomal protein $\mathrm{S} 7 \mathrm{p} / \mathrm{S} 5 \mathrm{e}$ (gene-C4D60_Mb00t01000) had lower expression MT with a $\log 2$ fold change value of -5.149 (Additional Table 3).

\section{Validation of DEGs by quantitative RT-PCR}

In order to confirm the validity of the RNA-Seq based transcript abundance of genes, a qRT-PCR analysis of five DEGs was performed; Ma10_g08300 (membrane transport proteins), Ma03_g32580 (TCP family transcription factor), Ma07_g22600 (probable mannan synthase 11 isoform X1), Ma06_g04390 (gibberellinregulated protein 6), and Ma08_g05160 (cellulose synthase-like protein). The Actin and Histone genes were used as internal controls. The qRT-PCR profiles of the five selected genes matched with the RNA-seq based results, which validates the useability of the Illumina sequencing results (Fig. 3).

\section{Differentially accumulated metabolites between WT and MT}

We also studied the WT and MT banana pseudostem metabolome to understand the role of metabolites in stem elongation. In total, we detected 412 metabolites. These detected metabolites could be grouped into 10 major classes (Additional Table 4). For the evaluation of differences in metabolite ion intensity between WT and MT, we applied the PLS-DA model. The established PLS-DA model showed good fitness (Additional Fig. 4).
Forty-eight metabolites were differentially accumulated in WT vs MT Aifen no. 1 banana pseudostem (Additional Fig. 5; Additional Table 5). Seventeen metabolites belonging to six classes i.e. amino acid and its derivatives (1), flavonoids (5), lignans and coumarins (4), organic acids (1), phenolic acids (3), and others (3), were higher in MT. The down-accumulated metabolites belonged to class alkaloids, amino acid and its derivatives, flavonoids, lignans and coumarins, lipids, nucleotide and its derivatives, organic acids, phenolic acids, tannins, terpenes, and others. The hierarchical clustering clearly differentiated the metabolites (Additional Fig. 6). The top-10 down-accumulated metabolites in MT were Asiatic acid (pmn001708), hydroxyanigorufone (XJ420P5484), cimidahurinine (pmn001553), coumarin (mws1012), isochlorogenic acid C (pmn001384), lumazine (mws1641), fer-agmatine (GQ512003), Nphenylacetylglycine (pme2743), 3-hydroxy-4-isopropylbenzylalcohol 3-glucoside (pmn001690), and plantainoside A (pmn001409). The top-10 up-regulated metabolites in MT were D-Xylonic acid lithium salt (mws0344), scutellarin (pmp000012), pinoresinol-hex (Qingke_Rfmb257-der01-3), pinoresinol-aceGlu (Qingke Rfmb257-der14-2), tricin O-saccharic acid (pmb3041), terpineol monoglucoside (pmn001378), inositol (pme0516), tricin 5-O-hexoside (pmb3042), sudachiin B (pmp001081), and sudachiin C (pmp001082) (Fig. 4a). We further functionally annotated the differentially accumulated metabolites (DAMs) in KEGG database and found that the most significantly enriched pathways were phenylalanine metabolism, phenylpropanoid biosynthesis, and tyrosine metabolism (Fig. 4b).

\section{Combined Transcriptome and Metabolome analyses}

To establish a relationship between different levels of molecules i.e. transcriptome and metabolome, we first visually compared the separate results of PCA. The PC1 of DEGs and DAMs had 23.07 and $26.11 \%$ variation, respectively. The PC2 of DEGs and DAMs showed 36.41 and $37.51 \%$ variation, respectively, suggesting that the
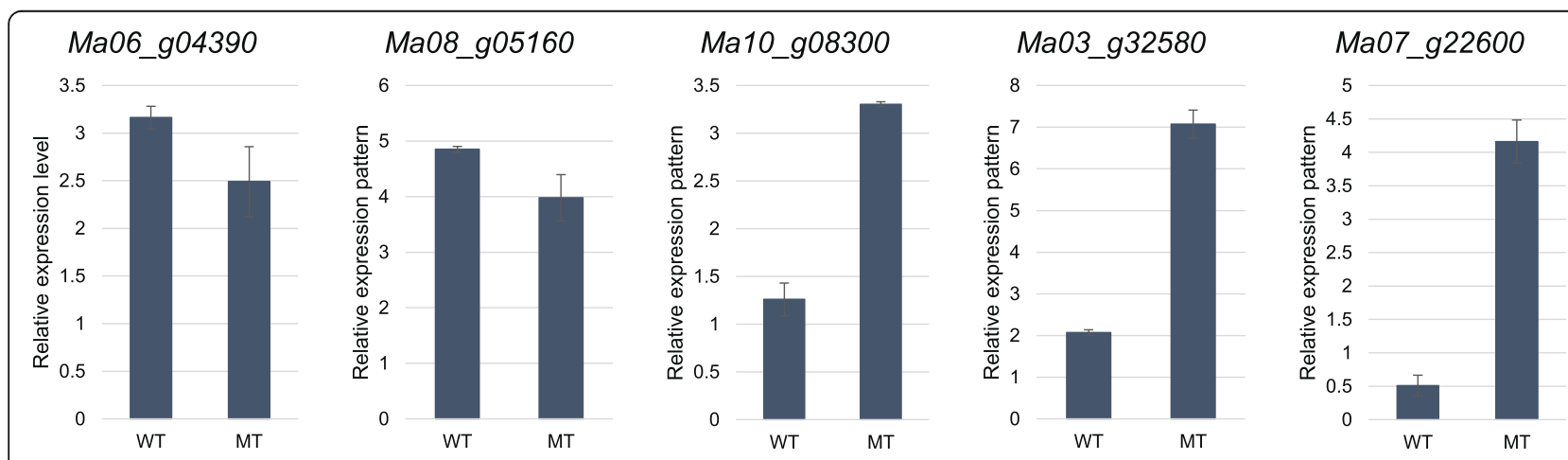

Fig. 3 qRT-PCR analysis of the five selected differentially expressed genes in WT and WT banana pseudostems 


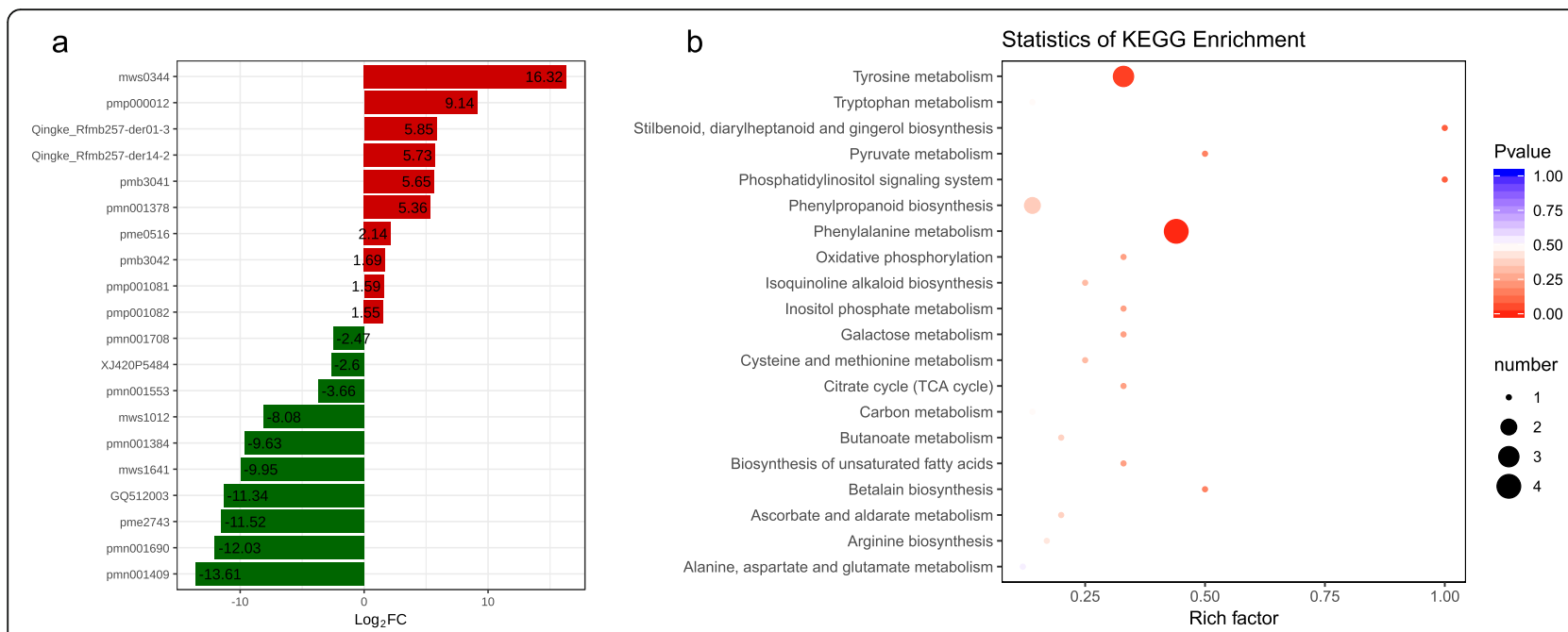

Fig. 4 a Top-10 up-accumulated (shown in red bars) and top-10 down-accumulated metabolites (shown in green bars) in WT vs MT Aifen No.1 banana pseudostem. $\mathbf{b}$ Scatter plot of 20 KEGG pathways between WT and MT Aifen No. 1 banana pseudostems to which the differentially accumulated metabolites were enriched. The degree of enrichment is shown by Rich factor, $P$-value, and the number of metabolites enriched in each pathway

amount of variation was more or less same (Additional Fig. 7). The co-joint KEGG pathway analysis showed that the DEGs and DAMs were mapped onto fifteen pathways (Additional Table 6; Fig. 5a). The co-joint KEGG pathway enrichment analysis showed that the same pathways were enriched as of transcriptome or metabolome based individual enrichment analysis. The significantly enriched pathways are shown in Fig. 5a.
Log2 conversion data for the DAMs and DEGs were selected that had a PCC $>0.8$. We then generated ninequadrant diagrams to visualize corresponding variations between DAMs and DEGs having PCC as given above (Fig. 5b; Additional Fig. 8). Transcript-metabolite correlation was determined by constructing subnetworks. These networks revealed both the regulatory and synthetic characteristics of genes and metabolites

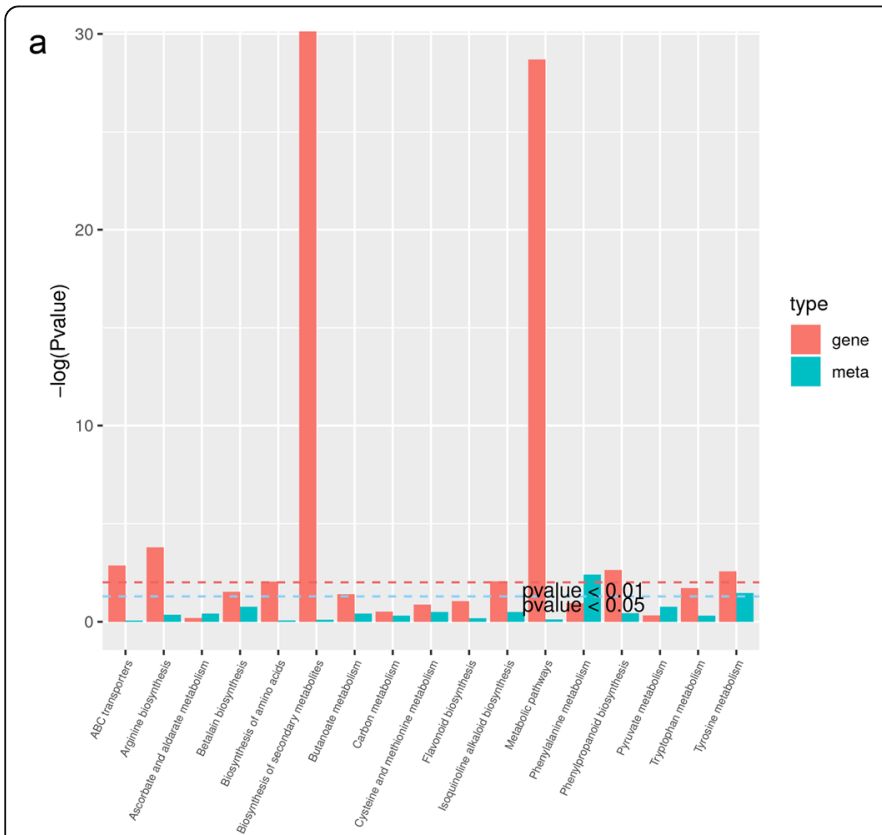

b

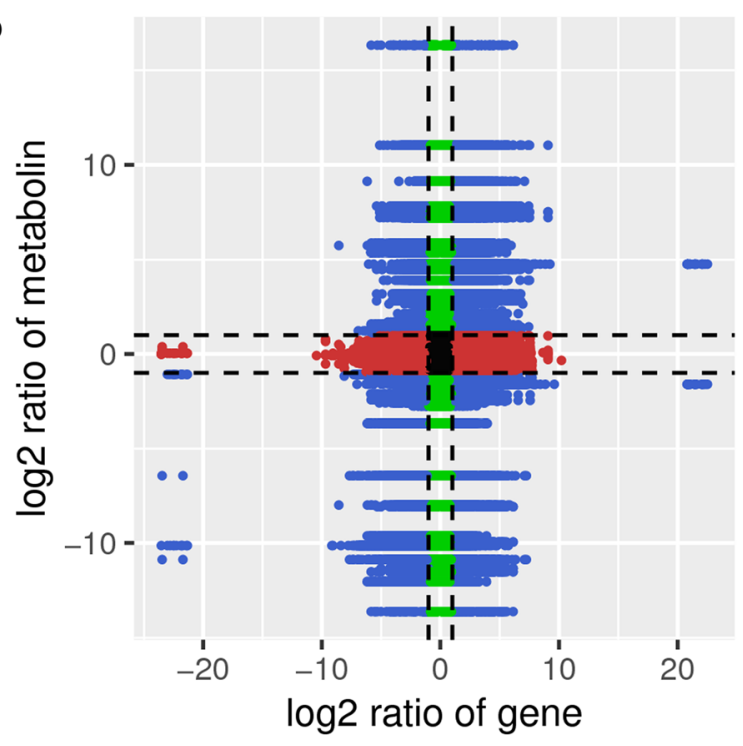

Fig. 5 Joint analysis of DEGs and DAMs between WT and MT Aifen No. 1 banana pseudostems. a Joint KEGG enrichment p-value histogram, $\mathbf{b}$ Pearson correlation coefficient of DEGs and DAMs in WT vs MT banana pseudostem represented as a nine-quadrant diagram. Black dots= unchanged genes/metabolites, green dots = DAMs with unchanged genes, red dots =DEGs with unchanged metabolites, blue dots $=$ DEGs and DAMs. The Pearson's correlation coefficient is $>0.8$ throughout the nine-quadrant graph 
(Additional Fig. 9). The correlation networks showed that transcripts and metabolites were correlated in four pathways i.e., Ascorbate and aldarate metabolism, cysteine and methionine metabolism, phenylpropanoid biosynthesis, and biosynthesis of amino acids. Furthermore, the relatively higher number of DAMs and DEGs in the 3rd and 7th quadrant indicates that the differential expression pattern of genes and differential accumulation of metabolites are consistent; genes and metabolites have a positive correlation, and the changes in metabolites may be positively regulated by genes (Fig. $5 \mathrm{a}$ ).

\section{Discussion}

Since the early banana improvement breeding efforts through tissue culture, dwarfism and stem height variants have been commonly observed [5]. Remarkable advances have been made to understand the mechanisms governing plant height in banana as well as other fruit trees. However, stem height/elongation is a complex physiological and biological process that involves various biological pathways (GA metabolism, BR, ABA, and auxin), mechanical properties of cell-walls, and coordinated signaling between the growing cells/tissues [3]. Here, we studied the DEGs and DAMs based on combined transcriptome and metabolome profiling of WT and MT of Pisang Awak Aifen No.1 for insights into the mechanisms related to banana plant height. We discuss the main pathways to which the studied DEGs and DAMs were associated.

It is known through loss-of-function mutations in the key GA biosynthesis regulatory enzymes display dwarf phenotypes [51]. Apart from biosynthesis-related genes, DELLAs are the key genes in the GA signal transduction pathway and DELLA deletion mutations in maize and brassica have been associated with dwarfism [52, 53]. The differential expression of DELLAs was not recorded in the comparative transcriptome analysis between WT and MT banana pseudostems. Similar observations have been previously recorded in autotetraploid apple where authors did not find differential regulation of DELLAs. However, the authors concluded that the stem height was being regulated by GA [54]. We also propose similar GA regulated pseudostem height regulation in MT. This proposition is based on many observations. First, the 36.66 fold up-regulation of one gibberellin regulated protein (Ma06_g04390) is an important observation, as in cucumber hypocotyl, this gene has been reported to encode a classical arabinogalactan protein and is involved in stem elongation [55]. Second, we also observed an increased expression of two other genes that are induced by GA i.e. interleukin-1 receptor-associated kinases with very high fold change values. This observation is in agreement with the fact that in rice, a receptor-like kinase named PSRK2 was induced by GA and was only found in the stem [56]. Third, the observation that a probable 2-oxoglutarate-dependent dioxygenase (Ma07_g15430) showed increased expression, further supports our proposition of possible involvement of GA in pseudostem height. This gene belongs to 2OG-Fe (II) oxygenase superfamily and its homologs are known for their role in catalyzation of latter reactions in the GA biosynthesis pathway [9]. Fourth, it is known that brassinosteroids (BRs) and GA are two predominant hormones regulating plant cell elongation. A defect in either of these leads to reduced plant growth and dwarfism. BRI is the major receptor of BR and overexpression of BRI1 in Arabidopsis has shown increased cell elongation and increased number of brassinolide binding sites in membrane fractions [57]. The significant increase in the expression of two BRI1 genes in MT suggests that BR is increasing pseudostem height by cell elongation. This observation is consistent with the results that decreased BR content in the autotetraploid apple caused dwarf phenotype [54]. Furthermore, BR catabolism is activated by LOB domain-containing proteins which are particularly important in the control of stem elongation [58]. We observed that one LOB domain-containing gene had higher expression in WT. LOB domaincontaining genes are targeted by DELLA proteins. LOB genes are categorized as organ boundary genes. Thus the organ boundary genes could regulate stem growth by restricting signals that control stem elongation, such as BR, which further regulates GA synthesis [28]. It has been already established in rice that BR modulates GA biosynthesis and hence regulates cell elongation. Fifth, the regulation of JAZ and TCP further supports the possible involvement of $\mathrm{GA}$ in pseudostem height regulation. Finally, the mevalonate pathway has also contribution to the GA biosynthesis pathway because geranylgeranyl diphosphate is synthesized by either a mevalonate-dependent or a non-mevalonate pathway [10]. The increased expression of a gene associated with mevalonate kinase activity suggests that GA metabolism is influencing the phenotype and the GA biosynthesis is possibly due to changes in the mevalonate-dependent pathway [9].

GAs also work in coordination with BR, ABA, auxin, and other hormones for stem growth regulation $[59,60]$. Auxin biosynthesis, transport, and sensitivity are associated with cell elongation in plants [61, 62]. Auxin has a specific role in epidermis during hypocotyl elongation is and controls BR synthesis and signaling [63]. Epidermis coordinates auxin-induced stem growth hence changes in auxin flux in cells could be linked with cell elongation via auxin efflux carrier proteins (also known as PINs) $[14,16]$. The up-regulation of two PINs in MT suggests that a similar mechanism might be present in banana stem. This observation is supported with the fact that 
we noticed up-regulation of one HD-zip protein in MT. Previously; links have also been established between auxin and the members of HD-zip proteins. The HD-zip gene (AtHB2) controls the elongation of hypocotyl depending on the auxin transport system $[64,65]$. Furthermore, $A B C$ transporters also play roles in auxin transport. The dwarf phenotype in Arabidopsis has also been implicated to $A B C$ transporters owing to their involvement in auxin transport [33]. Therefore, any upregulation of $\mathrm{ABC}$ transporter in $\mathrm{MT}$ will further verify that auxin influx in elongated pseudostem could be a possible regulatory mechanism. In this study two the $A B C$ transporter had higher expression. In addition, $\mathrm{ABC}$ transporter was one of the significantly enriched pathways in transcriptome as well as metabolome (Fig. 2b). Additionally, the relatively higher expression of phospholipases and a patatin-like protein 2 is quite relevant to these results because it has been established that phospholipases (including patatin-related phospholipases) have functions in auxin signal transduction [66]. Together, these findings suggest that auxin flux in the pseudostem was significantly affected and could lead to elongation of the stem.

Phospholipase-As have not been well characterized regarding cell elongation in Arabidopsis [67]. Therefore, the higher expression of phospholipases also suggests possible role in cell elongation in MT. Stem elongation or plant height is accomplished by cell expansion/elongation, which is a tightly regulated process. In Arabidopsis, cell elongation is further responsible for hypocotyl growth, while in cotton it is attributed to fiber elongation. The exclusive accumulation of scutellarin (pmp000012) in MT suggested that MT pseudostems are experiencing elongation. Scutellarin is a parahormone that promotes root elongation [68] (Additional Table 5). Overall, all plant organs owe their final size to a period of significant cell elongation [69]. Regarding cell elongation, our results showed that a probable GABA transporter had a lower expression, while a white-brown complex homolog protein 15 showed increased expression. These observations indicate that like Arabidopsis and cotton, banana cell elongation is also regulated in a complex manner $[45,46]$. As the size of a plant cell is determined by the extent of the surface of its wall, cell elongation may be defined also as any permanent increase in the surface of the cell wall [70]. Therefore, cell wall modification is an important consideration when comparing WT and MT banana pseudostems. We further checked whether cell-wall elasticity or porosity is affected during pseudostem elongation in banana. Previous investigations in Arabidopsis showed that cellulose composition and cell-wall thickness were affected very little during stem elongation. The role of pectin and cellulose is quite established in control of internode elongation [38]. Our observations in MT pseudostem suggest that banana might adopt a strategy to control the cell-wall rigidity and possibly reduce its viscoelasticity by down-regulating PMEI. The increased expression of probable mannan synthases and reduced expression of cellulose-synthase-like protein might be associated with protein-mediated changes in cell-wall extensibility driven by the circadian clock which should be further validated by future studies (Additional Table 3) [71, 72]. Furthermore, the increased expression of four COMTs is consistent with the previous reports on high lignin content, mechanical strength, and stem strength in lodging resistant wheat $[39,40]$. In this regard, the 81.968 fold higher accumulation of D-xylonic acid was an important observation with no accumulation in WT (Additional Table 5). D-xylonic acid has been found as a predominant constituent of the hemicellulose in pomegranate thus suggesting that large-scale cell wall modification is underway in MT [73]. This is further evident from the 57 and 53 fold up-accumulation of major lignans i.e. pinoresinol-hex and pinoresinol-aceGlu, respectively, in MT (Additional Table 5) [74].

Based on our results and known roles of different hormones and their cross talk with TFs, we propose that interaction of BRs, auxins, and ERFs with GA metabolism and signaling is likely to be responsible for the increase in pseudostem height of MT Aifen No.1 banana (Fig. 6). This novel understanding opens new research avenues for the further in-depth investigation of the separate and interactive role of each specified hormone and associated proteins or TFs.

\section{Conclusions}

In this study, we aimed at delineating possible mechanisms governing pseudostem height in banana by employing a combined transcriptome and metabolome analysis of a mutant and its wild type plant. At the metabolic level, phenylpropanoid biosynthesis, tyrosine metabolism, and phenylalanine metabolism pathways showed differential regulation. At the transcriptome level, we observed that biosynthesis of secondary metabolites, metabolic pathways, ribosomes and tyrosine metabolism were significantly enriched. The higher expression of a gibberellin regulated protein, interleukin-1 receptor-associated kinases, a probable 2-oxoglutatrate-dependent dioxygenase, and BRI genes in MT coupled with the changes in the expression of genes associated with mevalonate pathway indicates that GA is an important contributor in pseudostem height regulation. Our results also confirmed the known coordinated role of GAs with BR, ABA, auxin, and other hormones for stem growth regulation. The pseudostem height in MT was also being regulated by the changes in the expression of cell-wall related genes, cell elongation, and expansion. 


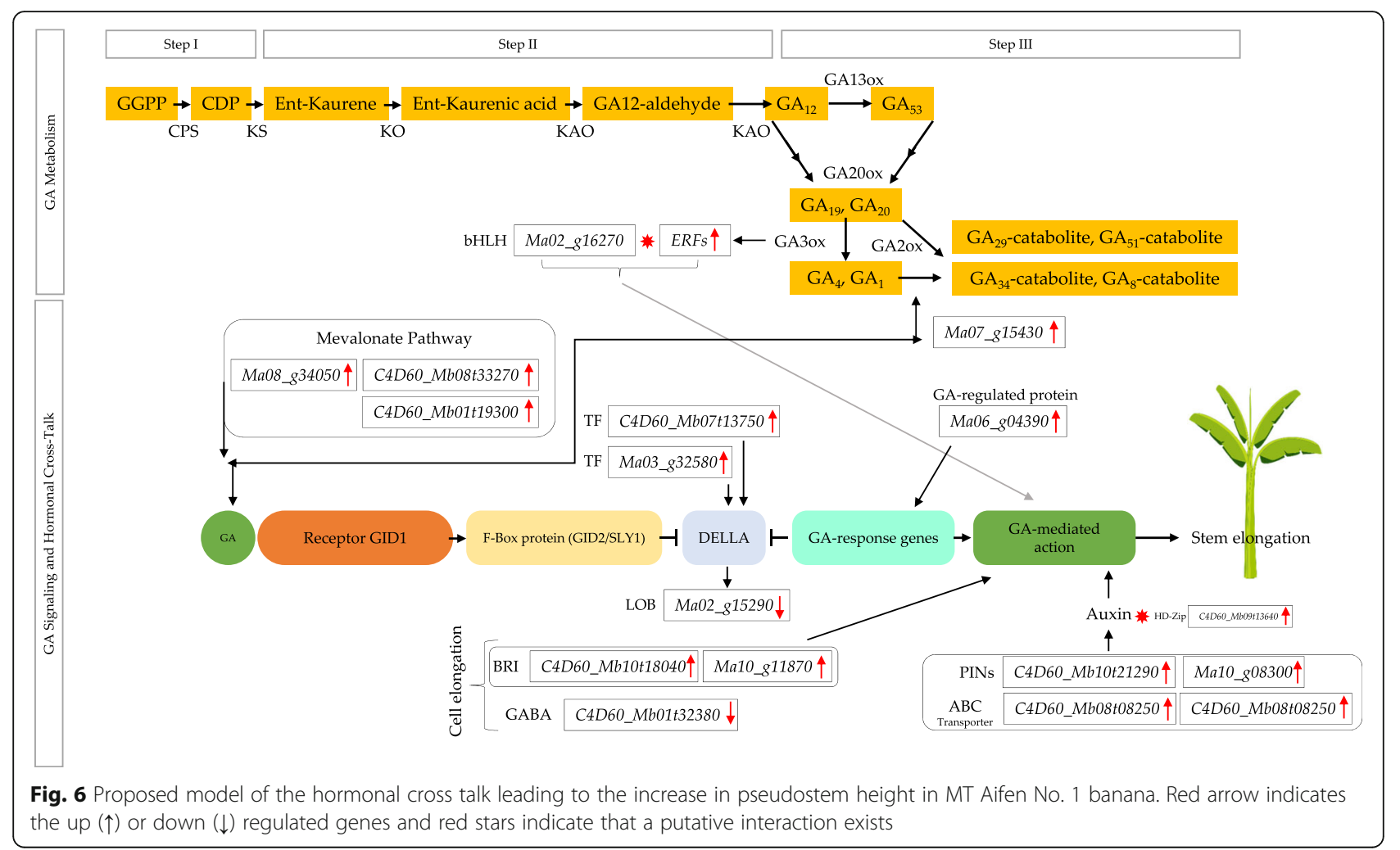

\section{Methods}

\section{Plant material}

The plant material was a wild type (WT) of a dwarf banana 'Aifen No. 1' (Musa spp. Pisang Awak subgroup $\mathrm{ABB}$ ) and its semi-dwarf mutant (MT). The MT was obtained from tissue-culture induced somaclonal variations in Aifen No. 1 WT at Institute of Fruit Tree Research, Guangdong Academy of Agricultural Sciences, Guangzhou, China by the corresponding author of this article (Professor Ou Sheng). The specimens used in this study have neither been reported nor submitted in any herbarium. The MT and WT were grown in nutrient-soil for four weeks of rooting and two days of hardening. After this, the seedlings were grown to reach five to six leaf stage ( 6 weeks old). The relative humidity, temperature, and photoperiod were $28^{\circ} \mathrm{C}, 60 \sim 80 \%$, and $12 \mathrm{~h}(1500 \pm 200 \mathrm{~lx})$, respectively. After six weeks, the banana plantlets had five to six true leaves. Triplicate pseudostems for WT as well as for MT were then cut and used for transcriptome and metabolome analyses (Fig. 1a).

\section{RNA extraction, library preparation, and Illumina Hiseq sequencing}

We used the standard procedures for the extraction of total RNAs from WT and MT pseudostems, which involved the use of Spin Column Plant total RNA Purification Kit (Tiandz, Beijing, China) [75]. Further, the
RNAs' purity was assessed, the extracted RNAs were quantified, and their integrity was checked. Following these steps, sequencing libraries were prepared and sequenced on the Illumina HiSeq platform (Illumina Inc., San Diego, USA) as reported earlier [76, 77].

\section{Sequencing data analysis}

We employed FastQC (http://www.bioinformatics. babraham.ac.uk/projects/fastqc/) to process the sequencing reads and obtain clean reads followed by determining the GC content distribution. HISAT2 was used to perform sequence alignment between clean reads and the reference genomes i.e. Musa genome (Musa acuminata DH Pahang V2 and Musa balbisiana DH PKW) $[18,27,78,79]$. The comparison efficiency (percentage of mapped reads to clean reads) was calculated followed by the calculation of the position distribution statistics of the reads on different chromosomes [80]. The regional distribution of the reads was compared and plotted as a chart. The results were visualized in IGV software [81]. The quality of the transcriptome library was assessed by randomization test of mRNA fragmentation followed by an insertion length check by calculating the distance between the start and end points of the reads on the reference genome at the ends of the insert. Gene expression was quantified as Fragments Per Kilobase of transcript per Million fragments mapped (FPKM). The gene expression data was then used to 
express the trend of gene abundance and overall distribution of gene expression, calculate correlation, and principal component analysis (PCA). For functional annotation, we aligned the genes with various databases such as KEGG [82], GO [83], Clusters of Orthologous Groups of proteins (COG) [84], and EuKaryotic Orthologous Groups (KOG) [85] using BLAST [86] with a threshold of E-value $<1.0 \times 10^{-5}$.

\section{Identification of differentially expressed genes, pathway enrichment, and real-time qRT-PCR}

The differential gene expression analysis and enrichment analyses were performed as previously described [76]. Briefly we used DESeq2 to differential expression genes (DEGs) after normalizing the read counts with the filtering criteria of fold change and false discovery rate (FDR) or $>2$ and $p<0.01$, respectively [87]. Hierarchical cluster analysis was done and represented as heat maps using Corset (https://code.google.com/p/corset-project/).

The identified DEGs were mapped on pathways in KEGG in KOBAS2.0 [88]. Care was taken to minimize the false positive prediction for which we employed FDR correction with $p<0.05$.

After analyzing the expression of genes, we selected five DEGs (Additional Table 7) that showed varied expression in WT and MT pseudostems and performed quantitative real-time PCR (qRT-PCR) on a Rotor-Gene 6000 machine (Qiagen, Shanghai, China) as following. cDNAs were synthesized from RNA using the High Capacity cDNA Reverse Transcription Kit (Applied Biosystem, MA, US). Two constitutively expressed genes i.e. Actin (F:CTCCTATGTTGCTCGC TTATG, R:GGCTACTACTTCGGTTCTTTC) and Histone H3.3 (F:GCGGTAATAGGAGTGAAGTC, R: TCAGCCTCAGCCAATGGCAC) were used as reference [89-91]. The temperature profiles, reagents, and volumes for PCR reactions were similar to previously described conditions [76, 92].

\section{Metabolome analysis}

Six weeks old pseudostems of the tissue cultured MT and WT Aifen No. 1 banana that were stored at $-80^{\circ} \mathrm{C}$ were processed at Wuhan MetWare Biotechnology Co., Ltd. (www.metware.cn). The remaining steps in processing the samples, extraction, and detection of the metabolites were done as reported earlier [93].

\section{Metabolomics data analysis}

The intensities of metabolites were submitted and processed in Analyst 1.6.1 software (AB SCIEX, Ontario, Canada) and metabolites with missing values were considered to be lower than the detection limit and replaced with a minimum recorded value. The ion intensities were normalized by log transformation, the metabolite abundance was calculated by using Dunnett's test, and multiple testing was controlled by FDR. To obtain the maximum differences between the treatments, we used the partial least squares-discriminant analysis (PLS-DA) and variable importance in projection $>1$ to screen DAMs was used to calculate the relative importance of the identified DAMs to the PLS-DA model. Other analyses including PCA, Hierarchical Clustering, and pathway enrichment were completed by using $\mathrm{R}$ (www.rproject.org) as reported earlier [20, 24, 82, 94].

\section{Combined Transcriptome and Metabolome analyses}

In addition to individual analyses for transcriptome sequencing and metabolome profiling, we performed cojoint analyses on DEGs and DAMs to determine the degree of enrichment of pathways. The Corson program in $\mathrm{R}$ was used to calculate the PCC of genes and metabolites and a correlation coefficient cluster heatmap of DEGs and DAMs was drawn. Gene-metabolite networks with a PCC $>0.8$ were used to construct the transcriptmetabolite network.

\section{Abbreviations}

GA: Gibbrellic acid; BR: Brassinosteroids; ABA: Abscisic acid; SAM: Shoot apical meristem; RZ: Rib zone; TF: Transcription factor; TCP: Class 1 teosinte branched 1; LOB: Lateral organ boundaries; WT: Wild type; MT: Mutant; FPKM: Fragments Per Kilobase of Transcript per Million Fragments Mapped; DEG: Differentially expressed; KEGG: Kyoto Encyclopedia of genes and Genomes; JAZ: Jasmonate ZIM domain-containing proteins; PSRK2: plant stature related receptor-like kinanse 2; GO: Gene Ontology; ERF: Ethylene responsive factor; GNAT: GCN5-related N-terminal acetyltransferases; PMEl: Pectin methylesterase inhibitors; COMT: Caffeic acid 3-Omethyltransferases; PLS-DA: Partial least squares-discriminant analysis; PCA: Principle component analysis; DAM: Differentially accumulated metabolites; PCC: Pearson correlation coefficients; COG: Clusters of Orthologous Groups of proteins; KOG: EuKaryotic Orthologous Groups; qRTPCR: Quantitative Real-time PCR

\section{Supplementary Information}

The online version contains supplementary material available at https://doi. org/10.1186/s12870-021-02899-6.

Additional file 1: Figure 1. Pearson correlation between WT vs MT banana pseudostem replicates. Figure 2. Heatmap hierarchical clustering of differential expressed genes in WT vs MT banana pseudostems where abscissa indicates the sample names (WT and MT), and the ordinate indicates the differential expressed genes. Figure 3. KEGG enrichment analysis of differentially expressed genes in WT vs MT banana pseudostems. Figure 4. Partial least squares-discriminant analysis. Figure 5. Variable importance in projection score plot. Figure 6. Heatmap hierarchical clustering of differentially accumulated metabolites. Figure 7. Principal Component Analysis (A) differentially expressed genes and (B) differentially accumulated metabolites. Figure 8. Correlation coefficient cluster heat map of differentially expressed genes and differentially accumulated metabolites having Pearson's correlation coefficient $>0.8$. Figure 9. Transcript-metabolite correlation network representing DAMs and DEGs involved in MT banana stem elongation. The KEGG pathways are given on the top of each network. The gene and metabolite IDs correspond to Additional Table 3 and Additional Table 5, respectively.

Additional file 2: Table 1: Summary of sequencing output statistics. Table 2: Mapping results of RNA-Seq data from WT and MT banana pseudostem. Table 3: Complete list of differentially expressed genes in 
WT vs MT banana pseudostem. Table 4: List of all detected metabolites in WT vs MT pseudostem. Table 5: List of differentially expressed metabolites in WT vs MT banana pseudostem. Table 6: Co-joint KEGG analysis. The list shows the pathways onto which the differentially regulated genes and differentially accumulated metabolites were mapped. Table $\mathbf{7}$ Primer sequences of the genes used for qRT-PCR.

\section{Acknowledgements}

Not applicable.

\section{Authors' contributions}

O. S., C.H. and G.Y. conceived the study and designed the experiments. G. D. F. B., J. L. and W. H. performed the experiments. O. S., C. L., X. Z. and G. D. were involved in funding acquisition. G. D., C. H., T. D., Q. Y. and H. G. analyzed the data. G. D., M. P. and T. D. were involved in obtaining the materials. G. D. and O. S. wrote the manuscript with contributions from the other authors. All authors have read and approved the final version of the manuscript.

\section{Funding}

This research was financially supported by National Key Research and Development Project (2018YFD1000102, 2019YFD1000200), Guangdong Science and Technology Project (2019B030316007), special fund for scientific innovation strategy-construction of high level Academy of Agriculture Science (R2018PY-QY004, R2017PY-QY001, R2017PY-JX002), Guangzhou national modern agricultural industry science and technology innovation center project (2018kczx06), and National Banana Industry and Technology System Project (CARS-31-01). The funder has no role in study design, data collection and analysis, decision to publish, or preparation of the manuscript.

\section{Availability of data and materials}

RNA-seq data is available at the SRA database in National Center of Biotechnology Information with the accession number PRJNA609266 (https://www.ncbi.nlm.nih.gov/sra/?term=PRJNA609266).

\section{Declarations}

\section{Ethics approval and consent to participate}

Not applicable.

\section{Consent for publication}

Not applicable.

\section{Competing interests}

The authors declare that they have no competing interests.

\section{Author details}

${ }^{1}$ Institute of Fruit Tree Research, Guangdong Academy of Agricultural Sciences, Guangzhou, China. ${ }^{2}$ Key Laboratory of South Subtropical Fruit Biology and Genetic Resource Utilization, Ministry of Agriculture, Guangzhou, China. ${ }^{3}$ Key Laboratory of Tropical and Subtropical Fruit Tree Research, Guangdong Province, Guangzhou, China. ${ }^{4}$ Horticulture and Landscape College, Hunan Agricultural University, Changsha 410128, China.

Received: 21 October 2020 Accepted: 21 February 2021 Published online: 01 March 2021

\section{References}

1. Chen J, Xie J, Duan Y, Hu H, Hu Y, Li W. Genome-wide identification and expression profiling reveal tissue-specific expression and differentiallyregulated genes involved in gibberellin metabolism between Williams banana and its dwarf mutant. BMC Plant Biol. 2016:16(1):123.

2. Peng J, Richards DE, Hartley NM, Murphy GP, Devos KM, Flintham JE, Beales J, Fish LJ, Worland AJ, Pelica F. 'Green revolution'genes encode mutant gibberellin response modulators. Nature. 1999;400(6741):256.

3. Serrano-Mislata A, Sablowski R. The pillars of land plants: new insights into stem development. Curr Opin Plant Biol. 2018;45:11-7.

4. Sandoval J, Kerbellec F, Cote F, Doumas P. Distribution of endogenous gibberellins in dwarf and giant off-types banana (Musa AAA, cv.'grand Nain') plants from in vitro propagation. Plant Growth Regul. 1995;17(3):219-24.
5. Côte F-X, Sandoval J, Marie P, Auboiron E. Variations in micropropagated bananas and plantains: literature survey. Fruits. 1993;48(1):15-23.

6. Hamill S, Doogan V, Smith M, Daniells J. Characterisation and early detection of an offtype from micropropagated lady finger bananas. Aust Exp Agric. 1999;39(8):1017-23.

7. De Beer Z, Sigawa A. Banana (Musa spp.) Juice Production in South Africa. In: IV International Symposium on Banana: International Conference on Banana and Plantain in Africa: Harnessing International 879: 2008; 2008. p. 233-8.

8. Hollender CA, Hadiarto T, Srinivasan C, Scorza R, Dardick C. A brachytic dwarfism trait ( $d w)$ in peach trees is caused by a nonsense mutation within the gibberellic acid receptor PpeGID1c. New Phytol. 2016;210(1):227-39.

9. Hedden P, Phillips AL. Gibberellin metabolism: new insights revealed by the genes. Trends Plant Sci. 2000;5(12):523-30.

10. T-p S. Gibberellin metabolism, perception and signaling pathways in Arabidopsis. In: The Arabidopsis Book/American Society of Plant Biologists; 2008. p. 6

11. Gaillochet C, Daum G, Lohmann JU. O cell, where art thou? The mechanisms of shoot meristem patterning. Curr Opin Plant Biol. 2015;23:91-7.

12. Davière J-M, Wild M, Regnault T, Baumberger $N$, Eisler $H$, Genschik P, Achard P. Class I TCP-DELLA interactions in inflorescence shoot apex determine plant height. Curr Biol. 2014;24(16):1923-8.

13. Bell EM, Lin W-c, Husbands AY, Yu L, Jaganatha V, Jablonska B, Mangeon A, Neff MM, Girke T, Springer PS. Arabidopsis lateral organ boundaries negatively regulates brassinosteroid accumulation to limit growth in organ boundaries. Proc Natl Acad Sci. 2012;109(51):21146-51.

14. Kleine-Vehn J, Leitner J, Zwiewka M, Sauer M, Abas L, Luschnig C, Friml J. Differential degradation of PIN2 auxin efflux carrier by retromer-dependent vacuolar targeting. Proc Natl Acad Sci. 2008;105(46):17812-7.

15. Nobusawa T, Okushima Y, Nagata N, Kojima M, Sakakibara H, Umeda M. Synthesis of very-long-chain fatty acids in the epidermis controls plant organ growth by restricting cell proliferation. PLoS Biol. 2013;11(4):e1001531.

16. Procko C, Burko Y, Jaillais $Y$, Ljung $K$, Long JA, Chory J. The epidermis coordinates auxin-induced stem growth in response to shade. Genes Dev. 2016;30(13):1529-41.

17. D'Hont A, Denoeud F, Aury J-M, Baurens F-C, Carreel F, Garsmeur O, Noel B, Bocs S, Droc G, Rouard M. The banana (Musa acuminata) genome and the evolution of monocotyledonous plants. Nature. 2012;488(7410):213.

18. Martin G, Baurens F-C, Droc G, Rouard M, Cenci A, Kilian A, Hastie A, Doležel J, Aury J-M, Alberti A. Improvement of the banana "Musa acuminata" reference sequence using NGS data and semi-automated bioinformatics methods. BMC Genomics. 2016;17(1):243.

19. Wang Z, Miao H, Liu J, Xu B, Yao X, Xu C, Zhao S, Fang X, Jia C, Wang J. Musa balbisiana genome reveals subgenome evolution and functional divergence. Nature plants. 2019;5(8):810-21.

20. Drapal M, de Carvalho EB, Rouard M, Amah D, Sardos J, Van den Houwe I, Brown A Roux N, Swennen R, Fraser PD. Metabolite profiling characterises chemotypes of Musa diploids and triploids at juvenile and pre-flowering growth stages. Sci Rep. 2019:9(1):4657.

21. Gamez RM, Rodríguez F, Vidal NM, Ramirez S, Alvarez RV, Landsman D, Mariño-Ramírez L. Banana (Musa acuminata) transcriptome profiling in response to rhizobacteria: bacillus amyloliquefaciens Bs006 and Pseudomonas fluorescens Ps006. BMC Genomics. 2019;20(1):378.

22. Sun J, Zhang J, Fang H, Peng L, Wei S, Li C, Zheng S, Lu J. Comparative transcriptome analysis reveals resistance-related genes and pathways in Musa acuminata banana'Guijiao 9'in response to Fusarium wilt. Plant Physiol Biochem. 2019;141:83-94

23. Xu M, Zeng C-B, He R, Yan Z, Qi Z, Xiong R, Cheng Y, Wei S-S, Tang H. Transcriptome Analysis of Banana (Musa acuminate L.) in Response to LowPotassium Stress. Agronomy. 2019;9(4):169.

24. Li T, Wu Q, Zhu H, Zhou Y, Jiang Y, Gao H, Yun Z. Comparative transcriptomic and metabolic analysis reveals the effect of melatonin on delaying anthracnose incidence upon postharvest banana fruit peel. BMC Plant Biol. 2019;19(1):289.

25. Nascimento TP, Castro-Alves VC, Castelan FP, Calhau MFN, Saraiva LA, Agopian RG, Cordenunsi-Lysenko BR. Metabolomic profiling reveals that natural biodiversity surrounding a banana crop may positively influence the nutritional/sensorial profile of ripe fruits. Food Res Int. 2019;124:165-74.

26. Yun Z, Li T, Gao H, Zhu H, Gupta VK, Jiang Y, Duan X. Integrated Transcriptomic, proteomic, and metabolomics analysis reveals Peel ripening of harvested Banana under natural condition. Biomolecules. 2019:9(5):167.

27. Rouard M, Droc G, Martin G, Sardos J, Hueber Y, Guignon V, Cenci A, Geigle B, Hibbins MS, Yahiaoui N. Three new genome assemblies support a rapid radiation in Musa acuminata (wild banana). Genome Biol Evol. 2018;10(12):3129-40. 
28. Tong H, Xiao Y, Liu D, Gao S, Liu L, Yin Y, Jin Y, Qian Q, Chu C. Brassinosteroid regulates cell elongation by modulating gibberellin metabolism in rice. Plant Cell. 2014;26(11):4376-93.

29. Baum G, Lev-Yadun S, Fridmann Y, Arazi T, Katsnelson H, Zik M, Fromm H. Calmodulin binding to glutamate decarboxylase is required for regulation of glutamate and GABA metabolism and normal development in plants. EMBO J. 1996;15(12):2988-96.

30. Fatland BL, Nikolau BJ, Wurtele ES. Reverse genetic characterization of cytosolic acetyl-CoA generation by ATP-citrate lyase in Arabidopsis. Plant Cell. 2005;17(1):182-203.

31. Boycheva I, Vassileva V, lantcheva A. Histone acetyltransferases in plant development and plasticity. Curr Genomics. 2014;15(1):28

32. Liu L, Tong H, Xiao Y, Che R, Xu F, Hu B, Liang C, Chu J, Li J, Chu C. Activation of big Grain1 significantly improves grain size by regulating auxin transport in rice. Proc Natl Acad Sci. 2015;112(35):11102-7.

33. Ye L, Liu L, Xing A, Kang D. Characterization of a dwarf mutant allele of Arabidopsis MDR-like ABC transporter AtPGP1 gene. Biochem Biophys Res Commun. 2013;441(4):782-6.

34. Chung HS, Howe GA. A critical role for the TIFY motif in repression of jasmonate signaling by a stabilized splice variant of the JASMONATE ZIMdomain protein JAZ10 in Arabidopsis. Plant Cell. 2009;21(1):131-45.

35. Shyu C, Figueroa P, DePew CL, Cooke TF, Sheard LB, Moreno JE, Katsir L, Zheng N, Howe GA. JAZ8 lacks a canonical degron and has an EAR motif that mediates transcriptional repression of jasmonate responses in Arabidopsis. Plant Cell. 2012;24(2):536-50.

36. Rietz S, Dermendjiev G, Oppermann E, Tafesse FG, Effendi Y, Holk A, Parker JE, Teige M, Scherer GF. Roles of Arabidopsis patatin-related phospholipases a in root development are related to auxin responses and phosphate deficiency. Mol Plant. 2010;3(3):524-38.

37. Nawaz MA, Rehman HM, Imtiaz M, Baloch FS, Lee JD, Yang SH, Lee SI, Chung G. Systems Identification and Characterization of Cell Wall Reassembly and Degradation Related Genes in Glycine max (L.) Merill, a Bioenergy Legume. Sci Rep. 2017;7(1):10862.

38. Wormit A, Usadel B. The multifaceted role of pectin methylesterase inhibitors (PMEls). Int J Mol Sci. 2018;19(10):2878.

39. $\mathrm{Ma} \mathrm{Q}-\mathrm{H}$. The expression of caffeic acid 3-O-methyltransferase in two wheat genotypes differing in lodging resistance. J Exp Bot. 2009;60(9):2763-71.

40. Wang M, Zhu X, Wang K, Lu C, Luo M, Shan T, Zhang Z. A wheat caffeic acid 3-O-methyltransferase TaCOMT-3D positively contributes to both resistance to sharp eyespot disease and stem mechanical strength. Sci Rep. 2018;8(1):1-14.

41. Li J, Zhou W, Francisco P, Wong R, Zhang D, Smith SM. Inhibition of Arabidopsis chloroplast $\beta$-amylase BAM3 by maltotriose suggests a mechanism for the control of transitory leaf starch mobilisation. PLoS One. 2017; 12(2):e0172504

42. Bu R, Wang R, Wei Q, Hu H, Sun H, Song P, Yu Y, Liu Q, Zheng Z, Li T. Silencing of glycerol-3-phosphate acyltransferase 6 (GPAT6) gene using a newly established virus induced gene silencing (VIGS) system in cucumber alleviates autotoxicity mimicked by cinnamic acid (CA). Plant Soil. 2019; 438(1-2):329-46

43. Wang X, Guan Y, Zhang D, Dong X, Tian L, Qu LQ. A ß-ketoacyl-CoA synthase is involved in rice leaf cuticular wax synthesis and requires a CER2LIKE protein as a cofactor. Plant Physiol. 2017;173(2):944-55.

44. Farrow SC, Facchini PJ. Functional diversity of 2-oxoglutarate/Fe (II)dependent dioxygenases in plant metabolism. Front Plant Sci. 2014;5:524.

45. Renault H, El Amrani A, Palanivelu R, Updegraff EP, Yu A, Renou J-P, Preuss D, Bouchereau A, Deleu C. GABA accumulation causes cell elongation defects and a decrease in expression of genes encoding secreted and cell wall-related proteins in Arabidopsis thaliana. Plant Cell Physiol. 2011;52(5): 894-908.

46. Zhu Y-Q, Xu K-X, Luo B, Wang J-W, Chen X-Y. An ATP-binding cassette transporter GhWBC1 from elongating cotton fibers. Plant Physiol. 2003; 133(2):580-8.

47. Vanous A, Gardner C, Blanco M, Martin-Schwarze A, Lipka AE, Flint-Garcia S, Bohn M, Edwards J, Lübberstedt T. Association mapping of flowering and height traits in germplasm enhancement of maize doubled haploid (GEMDH) lines. Plant Genome. 2018;11(2). https://doi.org/10.3835/plantgenome2 017.09.0083.

48. Huang Y, Guo Y, Liu Y, Zhang F, Wang Z, Wang H, Wang F, Li D, Mao D, Luan S. 9-cis-Epoxycarotenoid dioxygenase 3 regulates plant growth and enhances multi-abiotic stress tolerance in rice. Front Plant Sci. 2018;9:162.
49. Iwamoto M, Baba-Kasai A, Kiyota S, Hara N, Takano M. ACO1, a gene for aminocyclopropane-1-carboxylate oxidase: effects on internode elongation at the heading stage in rice. Plant Cell Environ. 2010;33(5):805-15.

50. Zheng M, Wang Y, Liu X, Sun J, Wang Y, Xu Y, Lv J, Long W, Zhu X, Guo X. The RICE MINUTE-LIKE1 (RML1) gene, encoding a ribosomal large subunit protein $\mathrm{L} 3 \mathrm{~B}$, regulates leaf morphology and plant architecture in rice. J Exp Bot. 2016;67(11):3457-69

51. Otani M, Meguro S, Gondaira H, Hayashi M, Saito M, Han D-S, Inthima P, Supaibulwatana K, Mori S, Jikumaru Y. Overexpression of the gibberellin 2 oxidase gene from Torenia fournieri induces dwarf phenotypes in the liliaceous monocotyledon Tricyrtis sp. J Plant Physiol. 2013;170(16):1416-23.

52. Lawit SJ, Wych HM, Xu D, Kundu S, Tomes DT. Maize DELLA proteins dwarf plant8 and dwarf plant9 as modulators of plant development. Plant Cell Physiol. 2010;51(11):1854-68.

53. Liu C, Wang J, Huang T, Wang F, Yuan F, Cheng X, Zhang Y, Shi S, Wu J, Liu K. A missense mutation in the VHYNP motif of a DELLA protein causes a semi-dwarf mutant phenotype in Brassica napus. Theor Appl Genet. 2010; 121(2):249-58.

54. Ma Y, Xue H, Zhang L, Zhang F, Ou C, Wang F, Zhang Z. Involvement of auxin and brassinosteroid in dwarfism of autotetraploid apple (Malus $X$ domestica). Sci Rep. 2016;6:26719.

55. Park MH, Suzuki Y, Chono M, Knox JP, Yamaguchi I. CsAGP1, a gibberellinresponsive gene from cucumber hypocotyls, encodes a classical arabinogalactan protein and is involved in stem elongation. Plant Physiol. 2003;131(3):1450-9.

56. Li Y, Tang D, Li L, Zhao X, Lin J, Liu X. Plant Stature Related receptor-like Kinanse2 (PSRK2) acts as a factor that determines stem elongation toward gibberellins response in rice. Biosci. 2018;82(11):1931-41.

57. Wang Z-Y, Seto H, Fujioka S, Yoshida S, Chory J. BRI1 is a critical component of a plasma-membrane receptor for plant steroids. Nature. 2001;410(6826):380.

58. Singh AP, Savaldi-Goldstein S. Growth control: brassinosteroid activity gets context. J Exp Bot. 2015;66(4):1123-32.

59. Unterholzner SJ, Rozhon W, Papacek M, Ciomas J, Lange T, Kugler KG, Mayer KF, Sieberer T, Poppenberger B. Brassinosteroids are master regulators of gibberellin biosynthesis in Arabidopsis. Plant Cell. 2015;27(8):2261-72.

60. Best NB, Hartwig T, Budka J, Fujioka S, Johal G, Schulz B, Dilkes BP. nana plant2 encodes a maize ortholog of the Arabidopsis brassinosteroid biosynthesis gene DWARF1, identifying developmental interactions between brassinosteroids and gibberellins. Plant Physiol. 2016;171(4):2633-47.

61. Ma L, Li G. Auxin-dependent cell elongation during the shade avoidance response. Front Plant Sci. 2019;10:914.

62. Le B, Nawaz MA, Rehman HM, Le T, Yang SH, Golokhvast KS, Son E, Chung $\mathrm{G}$. Genome-wide characterization and expression pattern of auxin response factor (ARF) gene family in soybean and common bean. Genes Genomics. 2016:38(12):1165-78.

63. Reed JW, Wu M-F, Reeves PH, Hodgens C, Yadav V, Hayes S, Pierik R. Three auxin response factors promote hypocotyl elongation. Plant Physiol. 2018; 178(2):864-75.

64. Steindler C, Matteucci A, Sessa G, Weimar T, Ohgishi M, Aoyama T, Morelli G, Ruberti I. Shade avoidance responses are mediated by the ATHB-2 HD-zip protein, a negative regulator of gene expression. Development. 1999; 126(19):4235-45.

65. Turchi L, Baima S, Morelli G, Ruberti I. Interplay of HD-zip II and III transcription factors in auxin-regulated plant development. J Exp Bot. 2015; 66(16):5043-53.

66. Scherer GF, Labusch C, Effendi Y. Phospholipases and the network of auxin signal transduction with $\mathrm{ABP} 1$ and TIR1 as two receptors: a comprehensive and provocative model. Front Plant Sci. 2012;3:56.

67. Lee HY, Bahn SC, Kang Y-M, Lee KH, Kim HJ, Noh EK, Palta JP, Shin JS, Ryu SB. Secretory low molecular weight phospholipase A2 plays important roles in cell elongation and shoot gravitropism in Arabidopsis. Plant Cell. 2003;15(9):1990-2002.

68. Li Weiqi LY, Xudong Z, Faqing T. Parahormone promoting plant root elongation and application thereof; 2013.

69. Azpiroz R, Wu Y, LoCascio JC, Feldmann KA. An Arabidopsis brassinosteroid-dependent mutant is blocked in cell elongation. Plant Cell. 1998;10(2):219-30.

70. Heyn A. The physiology of cell elongation. Bot Rev. 1940;6(10):515.

71. Ivakov A, Flis A, Apelt F, Fünfgeld M, Scherer U, Stitt M, Kragler F, Vissenberg K, Persson S, Suslov D. Cellulose synthesis and cell expansion are regulated by different mechanisms in growing Arabidopsis hypocotyls. Plant Cell. 2017;29(6):1305-15. 
72. Nawaz MA, Lin X, Chan T-F, Imtiaz M, Rehman HM, Ali MA, Baloch FS, Atif RM, Yang SH, Chung G. Characterization of cellulose synthase a (CESA) gene family in Eudicots. Biochem Genet. 2019;57(2):248-72.

73. Qin G, Liu C, Li J, Qi Y, Gao Z, Zhang X, Yi X, Pan H, Ming R, Xu Y. Diversity of metabolite accumulation patterns in inner and outer seed coats of pomegranate: exploring their relationship with genetic mechanisms of seed coat development. Horticulture Res. 2020;7(1):1-14.

74. Huis R, Morreel K, Fliniaux O, Lucau-Danila A, Fénart S, Grec S, Neutelings G, Chabbert B, Mesnard F, Boerjan W. Natural hypolignification is associated with extensive oligolignol accumulation in flax stems. Plant Physiol. 2012;158(4):1893-915.

75. Lee SI, Muthusamy M, Nawaz MA, Hong JK, Lim M-H, Kim JA, Jeong M-J. Genome-wide analysis of spatiotemporal gene expression patternsduring floral organ development in Brassica rapa. Mol Genet Genom. 2019;294(6): 1403-20.

76. Chen L, Wu Q, He W, He T, Wu Q, Miao Y. Combined De Novo Transcriptome and Metabolome Analysis of Common Bean Response to Fusarium oxysporum f. sp. phaseoli Infection. Int J Mol Sci. 2019;20(24):6278.

77. Zhuang H, Lou Q, Liu H, Han H, Wang Q, Tang Z, Ma Y, Wang H. Differential regulation of Anthocyanins in green and purple turnips revealed by combined De novo Transcriptome and Metabolome analysis. Int J Mol Sci. 2019;20(18):4387

78. Chen Q, Han Y, Liu H, Wang X, Sun J, Zhao B, Li W, Tian J, Liang Y, Yan J. Genome-wide association analyses reveal the importance of alternative splicing in diversifying gene function and regulating phenotypic variation in maize. Plant Cell. 2018;30(7):1404-23.

79. Kim D, Paggi JM, Park C, Bennett C, Salzberg SL. Graph-based genome alignment and genotyping with HISAT2 and HISAT-genotype. Nat Biotechnol. 2019;37(8):907-15.

80. Gel B, Serra E. karyoploteR: an R/bioconductor package to plot customizable genomes displaying arbitrary data. Bioinformatics. 2017:33(19):3088-90

81. Thorvaldsdóttir H, Robinson JT, Mesirov JP. Integrative genomics viewer (IGV): high-performance genomics data visualization and exploration. Brief Bioinform. 2013;14(2):178-92.

82. Kanehisa M, Goto S, Kawashima S, Okuno Y, Hattori M. The KEGG resource for deciphering the genome. Nucleic Acids Res. 2004;32(suppl_ 1):D277-80

83. Ashburner M, Ball CA, Blake JA, Botstein D, Butler H, Cherry JM, Davis AP, Dolinski K, Dwight SS, Eppig JT, et al. Gene ontology: tool for the unification of biology. Nat Genet. 2000;25(1):25.

84. Tatusov RL, Galperin MY, Natale DA, Koonin EV. The COG database: a tool for fenome-scale analysis of protein functions and evolution. Nucleic Acids Res. 2000;28(1):33-6.

85. Koonin EV, Fedorova ND, Jackson JD, Jacobs AR, Krylov DM, Makarova KS, Mazumder R, Mekhedov SL, Nikolskaya AN, Rao BS. A comprehensive evolutionary classification of proteins encoded in complete eukaryotic genomes. Genome Biol. 2004;5(2):R7.

86. Altschul SF, Madden TL, Schäffer AA, Zhang J, Zhang Z, Miller W, Lipman DJ. Gapped BLAST and PSI-BLAST: a new generation of protein database search programs. Nucleic Acids Res. 1997;25(17):3389-402.

87. Anders S, McCarthy DJ, Chen Y, Okoniewski M, Smyth GK, Huber W, Robinson MD. Count-based differential expression analysis of RNA sequencing data using $R$ and bioconductor. Nat Protocol. 2013;8(9):1765.

88. Xie C, Mao X, Huang J, Ding Y, Wu J, Dong S, Kong L, Gao G, Li C-Y, Wei L. KOBAS 2.0: a web server for annotation and identification of enriched pathways and diseases. Nucleic Acids Res. 2011;39(suppl_2):W316-22.

89. Xue $R$, Wu J, Zhu Z, Wang L, Wang X, Wang S, Blair MW. Differentially expressed genes in resistant and susceptible common bean (Phaseolus vulgaris L.) genotypes in response to Fusarium oxysporum f. sp. phaseoli. PLoS One. 2015;10(6):e0127698.

90. Chen L, Zhong H-y, Kuang J-f, Li J-g, Lu W-j, Chen J-y. Validation of reference genes for RT-qPCR studies of gene expression in banana fruit under different experimental conditions. Planta. 2011;234(2):377.

91. Su W, Yuan Y, Zhang L, Jiang Y, Gan X, Bai Y, Peng J, Wu J, Liu Y, Lin S. Selection of the optimal reference genes for expression analyses in different materials of Eriobotrya japonica. Plant Methods. 2019;15(1):7.

92. Silvia Sebastiani M, Bagnaresi P, Sestili S, Biselli C, Zechini A, Orrù L, Cattivelli $L$, Ficcadenti N. Transcriptome analysis of the melon-Fusarium oxysporum $\mathrm{f}$. sp. melonis race 1.2 pathosystem in susceptible and resistant plants. Front Plant Sci. 2017:8:362
93. Ying $H$, Shi J, Zhang S, Pingcuo G, Wang S, Zhao F, Cui Y, Zeng X. Transcriptomic and metabolomic profiling provide novel insights into fruit development and flesh coloration in Prunus mira Koehne, a special wild peach species. BMC Plant Biol. 2019;19(1):463.

94. Yang X, Xia X, Zhang Z, Nong B, Zeng Y, Wu Y, Xiong F, Zhang Y, Liang H, Pan Y, Dai G, Deng G, Li D. Identification of anthocyanin biosynthesis genes in rice pericarp using PCAMP. Plant Biotechnol J. 2019;17(9):1700-2.

\section{Publisher's Note}

Springer Nature remains neutral with regard to jurisdictional claims in published maps and institutional affiliations.
Ready to submit your research? Choose BMC and benefit from:

- fast, convenient online submission

- thorough peer review by experienced researchers in your field

- rapid publication on acceptance

- support for research data, including large and complex data types

- gold Open Access which fosters wider collaboration and increased citations

- maximum visibility for your research: over $100 \mathrm{M}$ website views per year

At BMC, research is always in progress.

Learn more biomedcentral.com/submissions 